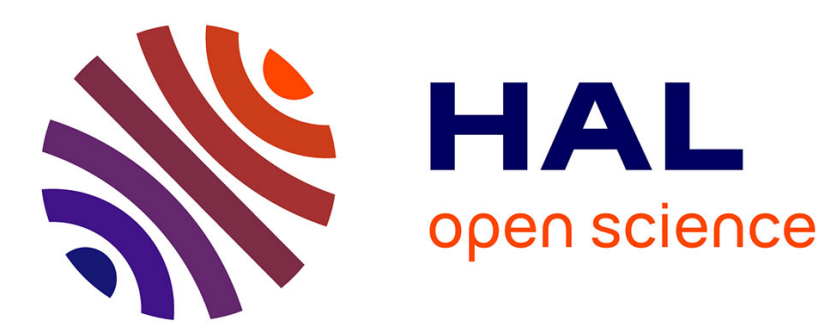

\title{
Expérimentation de rafraîchissement de l'espace public en période caniculaire à Paris
}

\author{
Martin Hendel, M Colombert, Y. Diab, L. Royon
}

\section{To cite this version:}

Martin Hendel, M Colombert, Y. Diab, L. Royon. Expérimentation de rafraîchissement de l'espace public en période caniculaire à Paris. Techniques Sciences Méthodes , 2015, 7-8, pp.41 - 51. 10.1051/tsm/201507041 . hal-01763633

\section{HAL Id: hal-01763633 \\ https://hal.science/hal-01763633}

Submitted on 11 Apr 2018

HAL is a multi-disciplinary open access archive for the deposit and dissemination of scientific research documents, whether they are published or not. The documents may come from teaching and research institutions in France or abroad, or from public or private research centers.
L'archive ouverte pluridisciplinaire HAL, est destinée au dépôt et à la diffusion de documents scientifiques de niveau recherche, publiés ou non, émanant des établissements d'enseignement et de recherche français ou étrangers, des laboratoires publics ou privés. 


\section{Cooling Public Spaces during Heat Wave Events in Paris}

\section{Expérimentation de rafraîchissement de l'espace public en période caniculaire à Paris}

M. HENDEL ${ }^{1,2,3}$, M. COLOMBERT ${ }^{2}$, Y. DIAB ${ }^{2}$, L.ROYON $^{3}$

\section{MOTS-CLES}

Ilot de chaleur urbain, adaptation au changement climatique, arrosage de l'espace public, confort thermique du piéton

\section{RESUME}

L'arrosage de l'espace public, couramment pratiqué du XIX $X^{\mathrm{e}}$ siècle jusqu'à la moitié du $\mathrm{XX}^{\mathrm{e}}$ siècle à Paris pour limiter les nuages de poussière connait un regain d'intérêt pour ses propriétés rafraîchissantes. Alors que les prévisions climatiques annoncent une augmentation en fréquence et en intensité des canicules en France, notamment en région parisienne, la méthode est perçue comme une mesure d'adaptation potentielle pour les milieux urbains denses. La Ville de Paris a conduit une expérimentation en 2013 pour continuer à étudier les effets de cette méthode, notamment thermiques et microclimatiques. L'analyse des mesures a montré des réductions de température de la chaussée pouvant atteindre $15^{\circ} \mathrm{C}$ à la surface ou $9^{\circ} \mathrm{C}$ à $5 \mathrm{~cm}$ de profondeur. Par ailleurs, l'absorption de chaleur par la chaussée à $5 \mathrm{~cm}$ de profondeur est réduite de plus de la moitié par l'arrosage. Enfin, les effets microclimatiques se soldent par un rafraîchissement statistiquement significatif, caractérisé par une réduction de température pouvant atteindre $0,7^{\circ} \mathrm{C}$ pour l'air et $1,8^{\circ} \mathrm{C}$ pour la température moyenne de rayonnement, en contrepartie d'une augmentation de l'humidité relative de moins de $3,7 \% \mathrm{HR}$.

\section{KEYWORDS}

Urban heat island, climate change adaptation, pavement-watering, pedestrian thermal comfort

\section{ABSTRACT}

Pavement-watering was used in Paris from the $19^{\text {th }}$ Century until the mid- $20^{\text {th }}$ Century against dust clouds. Today, its expected cooling properties are of growing interest. As climate change forecasts indicate increasing heat wave frequency and intensity for France and the Paris region, the method is viewed as a potential adaptation tool for dense urban areas. Paris City Hall conducted a pavementwatering experiment in 2013 to continue to study its thermal and micro-climatic effects. Results show pavement temperature reductions of up to $15^{\circ} \mathrm{C}$ at the surface and $9^{\circ} \mathrm{C}$ at a depth of $5 \mathrm{~cm}$. In addition, it was found that the heat absorption flux $5 \mathrm{~cm}$ deep in the pavement was more than halved during watering. Finally, the micro-climatic effects are found to result in statistically significant cooling, with up to $0.7^{\circ} \mathrm{C}$ and $1.8^{\circ} \mathrm{C}$ reductions in air and mean radiant temperatures, while relative humidity is increased by up to $3.7 \% \mathrm{RH}$.

\footnotetext{
${ }^{1}$ Mairie de Paris, Service Technique de l'Eau et de l'Assainissement, 27 rue du Commandeur, 75014 Paris, France

${ }^{2}$ Université Paris-Est, Lab'Urba, EA 843, EIVP, 80 rue Rébeval, 75019 Paris, France

${ }^{3}$ Univ Paris Diderot, Sorbonne Paris Cité, Laboratoire Matière et Systèmes Complexes, UMR 7057, CNRS, 10 rue Léonie Duquet, 75013 Paris, France
} 


\section{INTRODUCTION}

Bien que pratique courante contre les nuages de poussière à Paris du XIX ${ }^{e}$ siècle jusqu'à la première moitié du $X X^{e}$ siècle, l'arrosage de l'espace public suscite un nouvel intérêt comme méthode d'amélioration du confort thermique. Alors que les canicules accentuent les îlots de chaleur urbain (ICU) [1], il est prévu que leur fréquence et leur intensité augmentent au cours des années à venir en région parisienne [2]. Parallèlement, une diminution de la disponibilité estivale des eaux de surface est prévue [3]. Ainsi, les collectivités intéressées par cette méthode de rafraîchissement ont besoin d'une connaissance fiable de son efficacité et de son rendement.

A Paris, le réseau d'eau non potable, dont le maintien a été décidé le 19 mars 2012, a été identifié comme un outil particulièrement adapté pour l'adoption de cette pratique. Une méthode d'arrosage qui s'appuie sur ce réseau a ainsi été étudiée dans le cadre d'un projet de recherche (EPICEA) mené entre 2008 et 2012 par Météo-France et le CSTB, en partenariat avec l'atelier parisien de l'urbanisme (APUR) [4].

Suite à ces travaux, une première expérimentation a été menée pendant l'été 2012 dont les résultats ont été décrits par Bouvier et al. [5]. En cas de vague de chaleur, un arrosage nocturne était déclenché vers $22 \mathrm{~h}$ et l'évolution de la température et de l'humidité était suivie par des capteurs à différentes hauteurs. Sur quatre essais réalisés, le résultat maximum a été une diminution de $0,4^{\circ} \mathrm{C}$ de l'air et une augmentation de l'humidité de $3 \%$ à $2 \mathrm{~m}$ de hauteur pendant 1 heure environ après arrosage, accompagné d'une diminution des températures de surface de la chaussée de 4 à $6^{\circ} \mathrm{C}$.

Suite à ce travail, une nouvelle campagne expérimentale a été menée pendant l'été 2013 avec un protocole d'arrosage plus intensif pour améliorer la connaissance des performances de cette méthode. Nous discuterons ici des effets de l'arrosage sur le comportement thermique de la chaussée ainsi que de ses effets microclimatiques.

\section{MATERIELS ET METHODES}

\subsection{Choix des sites expérimentaux}

Les mesures ont été effectuées en continu durant l'été 2013 sur deux sites, comprenant une zone témoin et une zone arrosée équipées de station de mesure de température, d'humidité, de flux et vitesse du vent. Les positions des sites et stations sont illustrées à la Figure 1. La position de chaque station d'une même paire a été sélectionnée pour que l'environnement immédiat de chacune soit le plus similaire de celui de l'autre.

La rue du Louvre, qui traverse les $1^{\text {er }}$ et $2^{\mathrm{e}}$ Arrondissements, ainsi que les rues Lesage et Ramponeau, situées à proximité du parc de Belleville dans le $20^{\mathrm{e}}$ arrondissement, ont été sélectionnées pour les essais menés en 2013. 

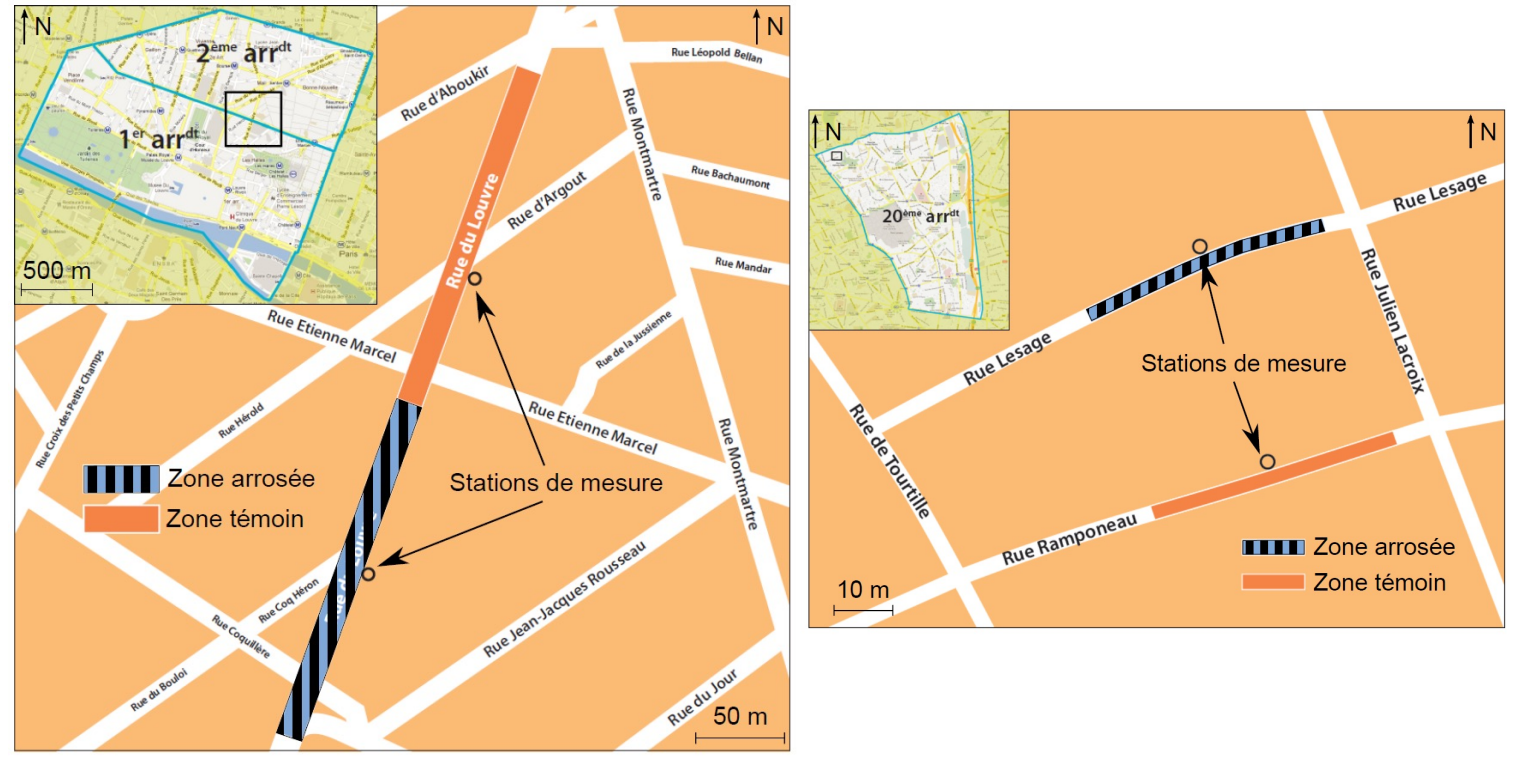

Figure 1 : Sites expérimentaux et positions des stations de mesure (à gauche : Louvre; à droite : Belleville)

\subsection{Méthode d'arrosage}

L'arrosage était déclenché si certaines conditions météorologiques étaient annoncées à trois jours par Météo-France. Quatre seuils devaient être atteints : 1) $\left.\left.I \mathrm{BM}^{4}{ }_{\operatorname{Min}}>16^{\circ} \mathrm{C}, 2\right) \mid \mathrm{IBM}_{\mathrm{Max}}>25^{\circ} \mathrm{C}, 3\right)$ vitesse du vent inférieure à $10 \mathrm{~km} / \mathrm{h}$ et 4) ciel non couvert. A Paris, l'alerte canicule est déclenchée pour des températures minimales et maximales supérieures à $21^{\circ}$ et $31^{\circ} \mathrm{C}$ pendant trois jours consécutifs, respectivement.

Rue du Louvre, l'aspersion d'eau était assurée par des laveuses qui apportaient environ $1 \mathrm{l} / \mathrm{m}^{2}$ toutes les heures entre $6 \mathrm{~h} 30$ et $11 \mathrm{~h} 30$ (soit $1 \mathrm{~mm} / \mathrm{h}$ ) et toutes les 30 minutes de $14 \mathrm{~h}$ à $18 \mathrm{~h} 30$ (soit $2 \mathrm{~mm} / \mathrm{h}$ ) sur le trottoir et la chaussée (cf. Figure 2). Les horaires de passage étaient notés par les chauffeurs.

Rue Lesage, un tuyau branché sur une bouche de lavage et tiré le long de la rue sur une quarantaine de mètres (cf. Figure 3 ) arrosait la chaussée de façon continue de $7 \mathrm{~h}$ à $19 \mathrm{~h}$. Un compteur a permis de suivre les consommations et débits d'eau $(25 \mathrm{~mm} / \mathrm{h})$.

L'eau utilisée pour ces essais est issue du réseau d'eau non potable de la Ville de Paris. Ce réseau est aujourd'hui considéré par les gestionnaires de la ville comme très prometteur pour le développement durable de Paris [6].

\footnotetext{
${ }^{4}$ IBM : Indice biométéorologique. Il s'agit de la moyenne de la température maximale ou minimale calculée sur 3 jours consécutifs.
} 


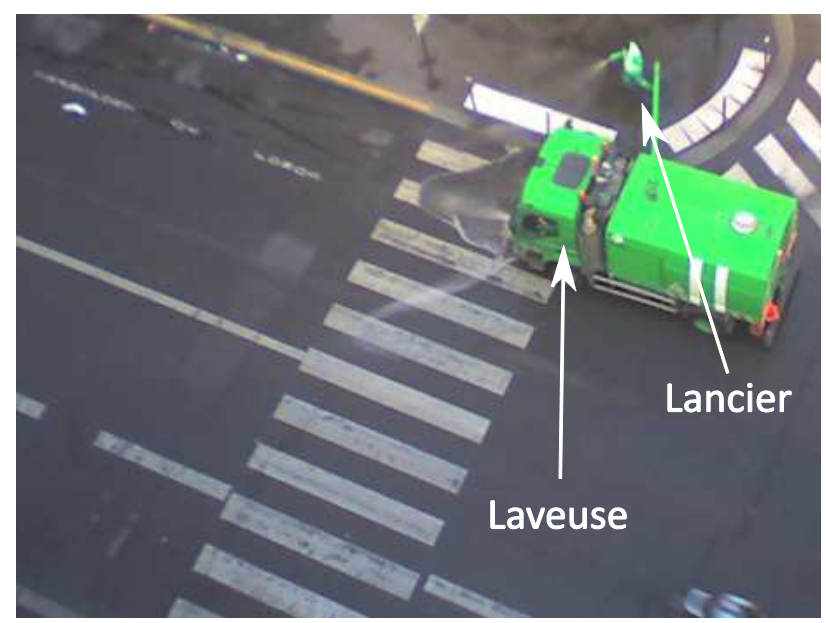

Figure 2 : Arrosage du trottoir et de la chaussée par laveuse et lancier de la rue du Louvre

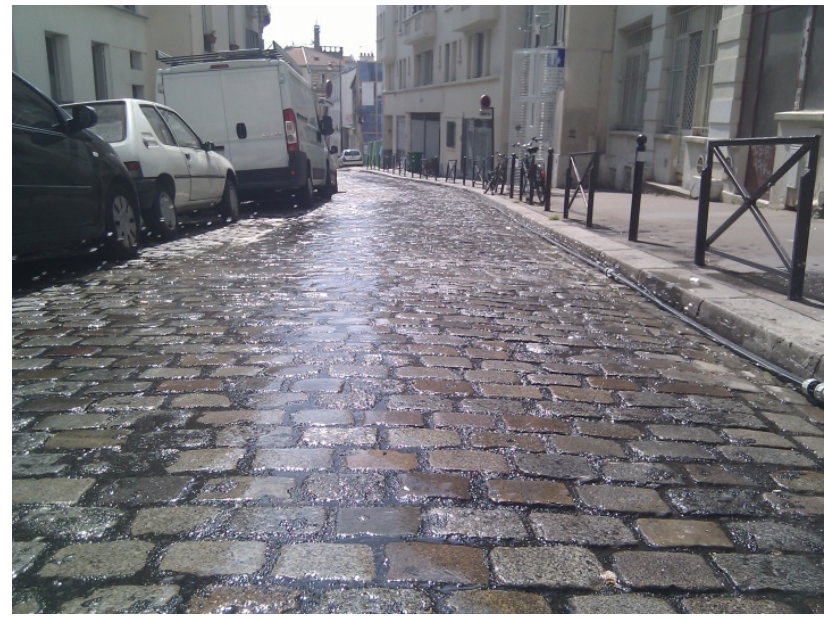

Figure 3 : Arrosage par tuyau percé de la rue Lesage

\subsection{Instrumentation}

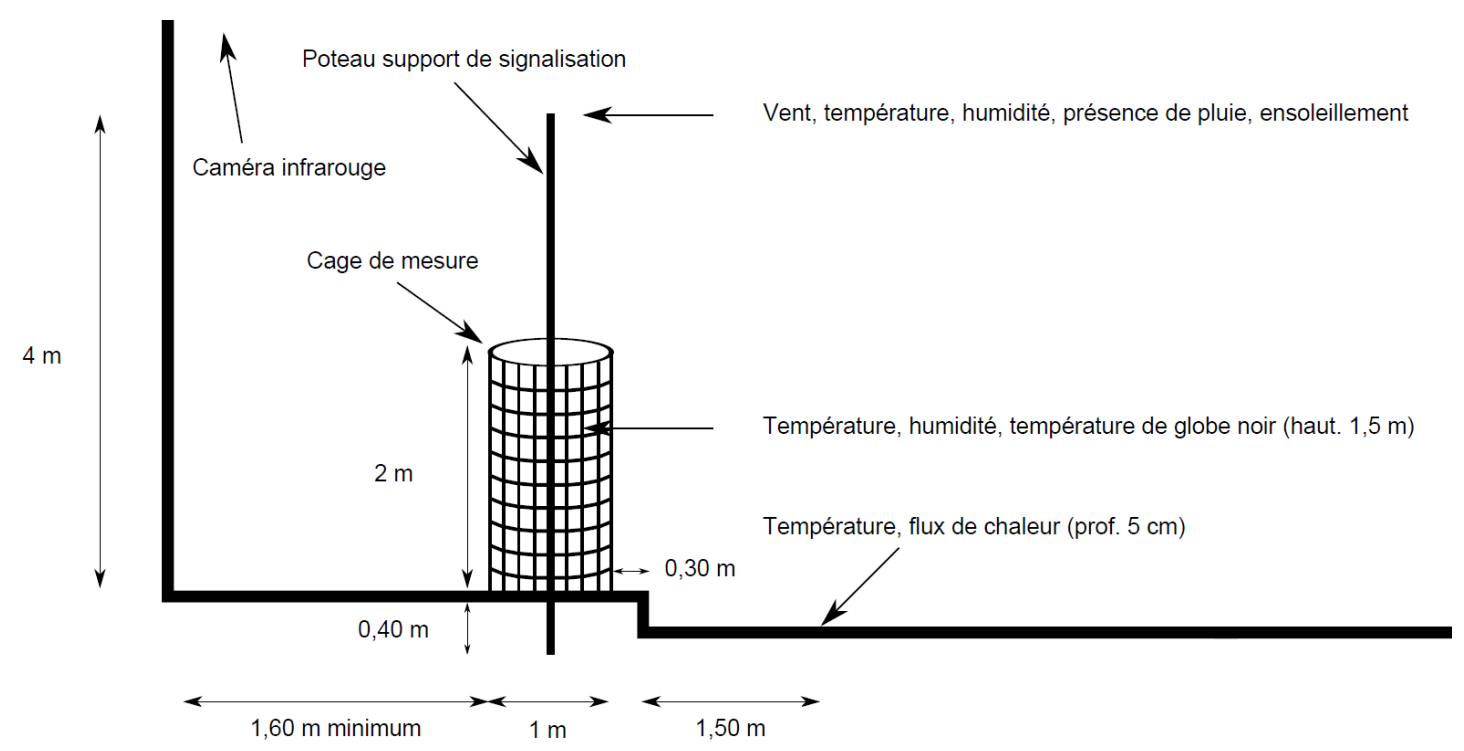

Figure 4 : Schéma de l'instrumentation

L'instrumentation des essais de l'été 2013 a fait appel à de nouveaux capteurs plus précis que ceux de 2012. La rue du Louvre a de plus été équipée de capteurs de flux et de température posés dans la chaussée à $5 \mathrm{~cm}$ de profondeur afin de pouvoir mieux caractériser son comportement thermique. Les 
instruments de mesures météorologiques ont été posés sur un mât de $4 \mathrm{~m}$ situé à proximité de la bordure de trottoir. Ceux à hauteur d'homme ont en outre été protégés par une cage cylindrique en acier. Un schéma des stations est présenté à la Figure 4.

Le fluxmètre, posé à $5 \mathrm{~cm}$ de profondeur dans la chaussée, mesure la chaleur transmise par conduction de l'environnement vers la chaussée. Ainsi, une mesure positive correspond à un stockage de chaleur (transfert du haut vers le bas), tandis qu'une mesure négative traduit une restitution de chaleur vers l'atmosphère (transfert du bas vers le haut).

Enfin, une caméra thermique fixe était posée sur la toiture-terrasse du bâtiment situé devant la station arrosée rue du Louvre et une caméra infrarouge prêtée par l'APUR a permis de réaliser des mesures mobiles des températures de surface.

Le Tableau 1 résume les instruments utilisés, leur hauteur et leur précision de mesure.

Tableau 1 : Instruments, hauteur et précision de mesure

\begin{tabular}{|c|c|c|c|}
\hline Paramètre & Instrument & Hauteur & Précision \\
\hline Ensoleillement & Pyranomètre de seconde classe - ISO 9060 & $4 \mathrm{~m}$ & $10 \%$ par jour \\
\hline Flux de chaleur chaussée & Fluxmètre sur mesure & $-5 \mathrm{~cm}$ & $5 \%$ \\
\hline Humidité relative & Hygromètre capacitive & $\begin{array}{c}1,5 \mathrm{~m} \\
4 \mathrm{~m}\end{array}$ & $1,5 \% \mathrm{HR}$ \\
\hline Température de l'air & Pt100 1/3 DIN B & $\begin{array}{c}1,5 \mathrm{~m} \\
4 \mathrm{~m}\end{array}$ & $0,1^{\circ} \mathrm{C}$ \\
\hline Température de globe noir & Pt100 1/2 DIN A - ISO 7726 & $1,5 \mathrm{~m}$ & $0,15^{\circ} \mathrm{C}$ \\
\hline Vitesse du vent & Anémomètre à ultrasons 2D & $4 \mathrm{~m}$ & $2 \%$ \\
\hline
\end{tabular}

Pour l'analyse des températures et du flux conductif seuls des mesures du site arrosé rue du Louvre sont disponibles, sauf pour les thermographies issues des campagnes mobiles. On procèdera donc par comparaison entre journées arrosées et journées sèches (témoins) aux conditions d'ensoleillement identiques ou presque. Pour les paramètres microclimatiques, une méthodologie spécifique est décrite plus loin.

\section{EFFETS THERMIQUES}

\subsection{Températures de surface}

La Figure 5 présente les températures de surface de la zone de chaussée située au-dessus du capteur de flux rue du Louvre. La courbe de gauche représente les mesures obtenues le 21 juillet, journée non arrosée, et celle de droite représente les mesures du 22 juillet, arrosée. Les points manquants sont dus à des véhicules garés ou passants au moment du cliché. Les droites en pointillés le 21 juillet indiquent le début (13h35) et la fin (18h30) de l'ensoleillement de la zone de chaussée étudiée. Pour le 22 juillet, les droites en tirets-pointillés représentent les arrosages de la chaussée.

En conditions sèches ou arrosées, la température de surface de la chaussée reste comprise entre $25^{\circ}$ et $55^{\circ} \mathrm{C}$ environ. En revanche, alors que l'allure globale les jours témoins est celle d'une courbe en cloche (perturbée le 21 juillet par des passages nuageux entre $14 \mathrm{~h}$ et $16 \mathrm{~h}$ ), celle-ci est fortement modifiée les jours arrosés. 

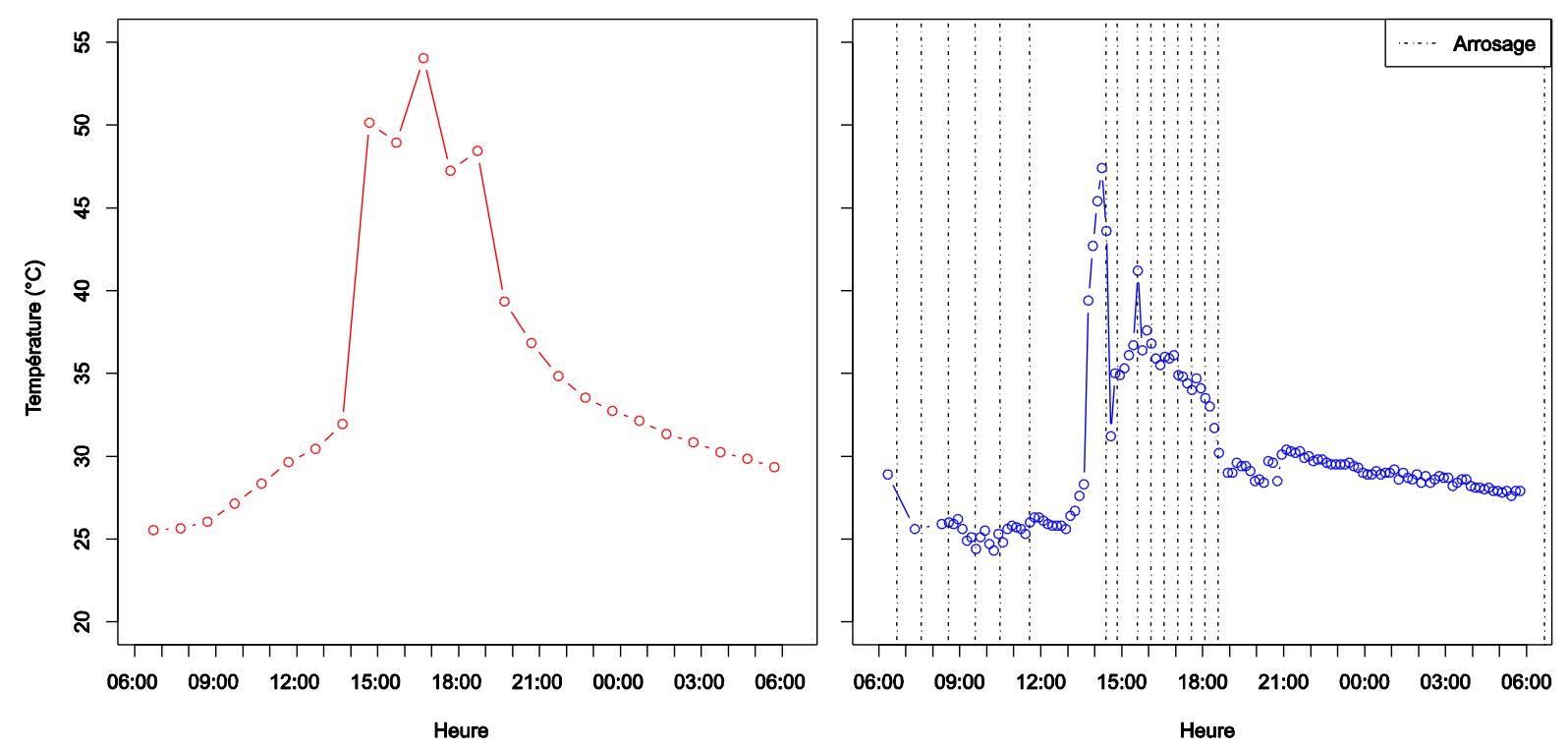

Figure 5 : Relevés des températures de surface apparentes corrigées de la chaussée rue du Louvre (à gauche : 21 juillet 2013 ; à droite : 22 juillet 2013)

L'arrosage provoque plusieurs bouleversements. Au premier arrosage matinal, une brusque chute de température de l'ordre de quelques degrés Celsius a lieu. Compte tenu des températures observées entre minuit et $6 \mathrm{~h} 30$, on estime que le rafraîchissement atteint $5^{\circ} \mathrm{C}$ environ. L'arrosage permet en plus de maintenir la température à sa valeur minimale pendant plusieurs heures et jusqu'à $1 \mathrm{~h} 30$ après la fin de l'arrosage matinal, alors que le minimum n'était que ponctuel le 21 juillet.

L'après-midi, une fois la chaussée au soleil, la température est environ $15^{\circ} \mathrm{C}$ plus fraîche entre $15 \mathrm{~h}$ et $18 \mathrm{~h} 30$ et le pic de température est atteint quelques minutes avant le premier arrosage de l'aprèsmidi. Un deuxième et dernier pic est observé entre $15 \mathrm{~h}$ et $16 \mathrm{~h}$, lors d'une longue interruption de l'arrosage.

Le soir, un " puits » est visible entre $18 \mathrm{~h} 30$ et $21 \mathrm{~h}$. Il est dû au passage à l'ombre suivi du séchage du support deux heures après le dernier arrosage. Enfin, la température après séchage vers $21 \mathrm{~h}$ est plus faible que le 21 juillet.

On constate immédiatement les effets de l'arrosage sur les températures de surface : température maximale et minimale réduites de plusieurs degrés, température minimale maintenue pendant plusieurs heures, rafraîchissement d'environ $15^{\circ} \mathrm{C}$ l'après-midi pendant l'ensoleillement.

Si un suivi aussi précis des températures de surface n'a pas pu être réalisé sur le site de Belleville, la Figure 6 montre des résultats similaires, au moins le soir. 


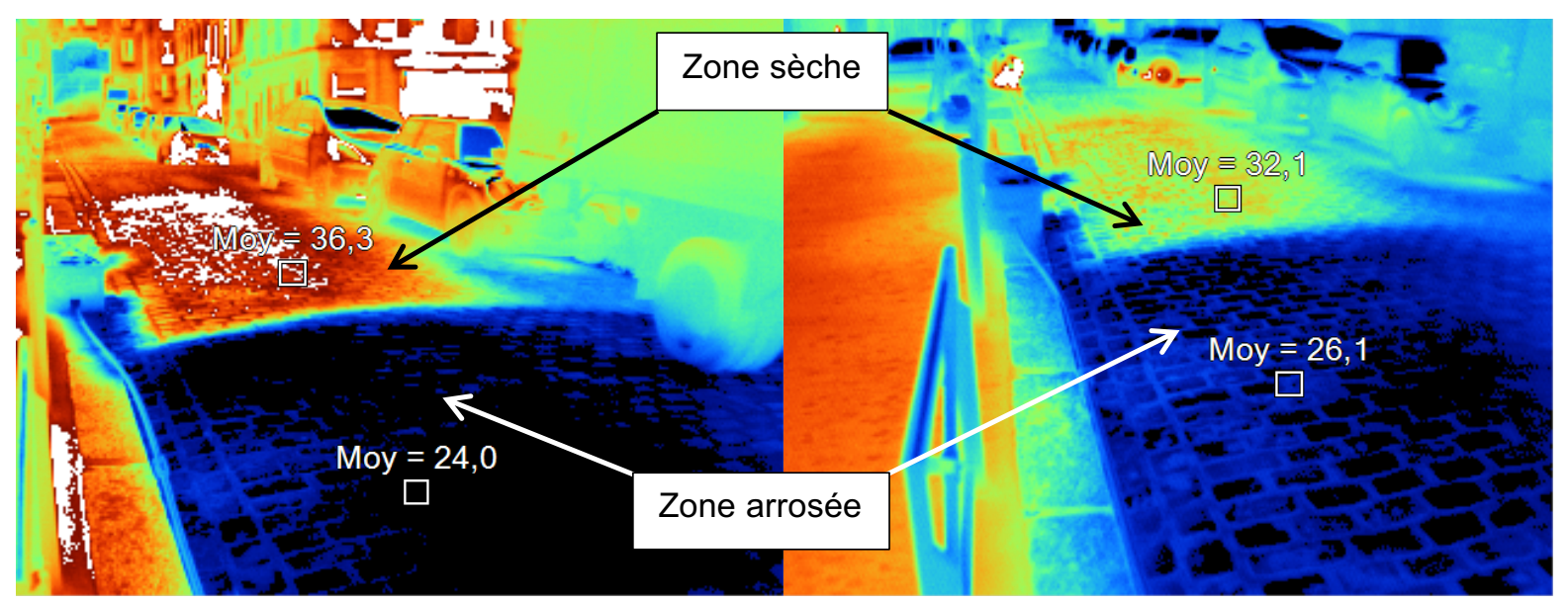

Figure 6 : Températures de surface apparentes $\left(\mathrm{en}^{\circ} \mathrm{C}\right.$ ) mesurées rue Lesage le 8 juillet 2013 (à gauche : 15 minutes après arrosage ; à droite : 3 heures après arrosage)

En effet, 15 minutes après arrosage, la zone arrosée est environ $10^{\circ} \mathrm{C}$ plus fraîche que la zone sèche. Cet écart se maintient plusieurs heures durant, étant encore de l'ordre de $6^{\circ} \mathrm{C}$ trois heures plus tard. De la même façon que pour la rue du Louvre, l'augmentation en température observée dans la zone arrosée est principalement due au séchage du support. En revanche, le trottoir, non arrosé et situé du côté Nord de la rue, est encore très chaud, passant de $37^{\circ} \mathrm{C}$ à $34^{\circ} \mathrm{C}$ environ sur la même période.

\subsection{Température à $\mathbf{5} \mathrm{cm}$ de profondeur}

La Figure 7 présente l'évolution de la température de la chaussée à $5 \mathrm{~cm}$ de profondeur mesurée par le capteur de chaussée les 21 et 22 juillet 2013. Comme pour la Figure 5, les droites verticales en tirets-pointillés le 22 juillet représentent les passages de la laveuse et les droites en pointillés du 21 juillet représentent le début et la fin de l'ensoleillement de la chaussée au-dessus du capteur.
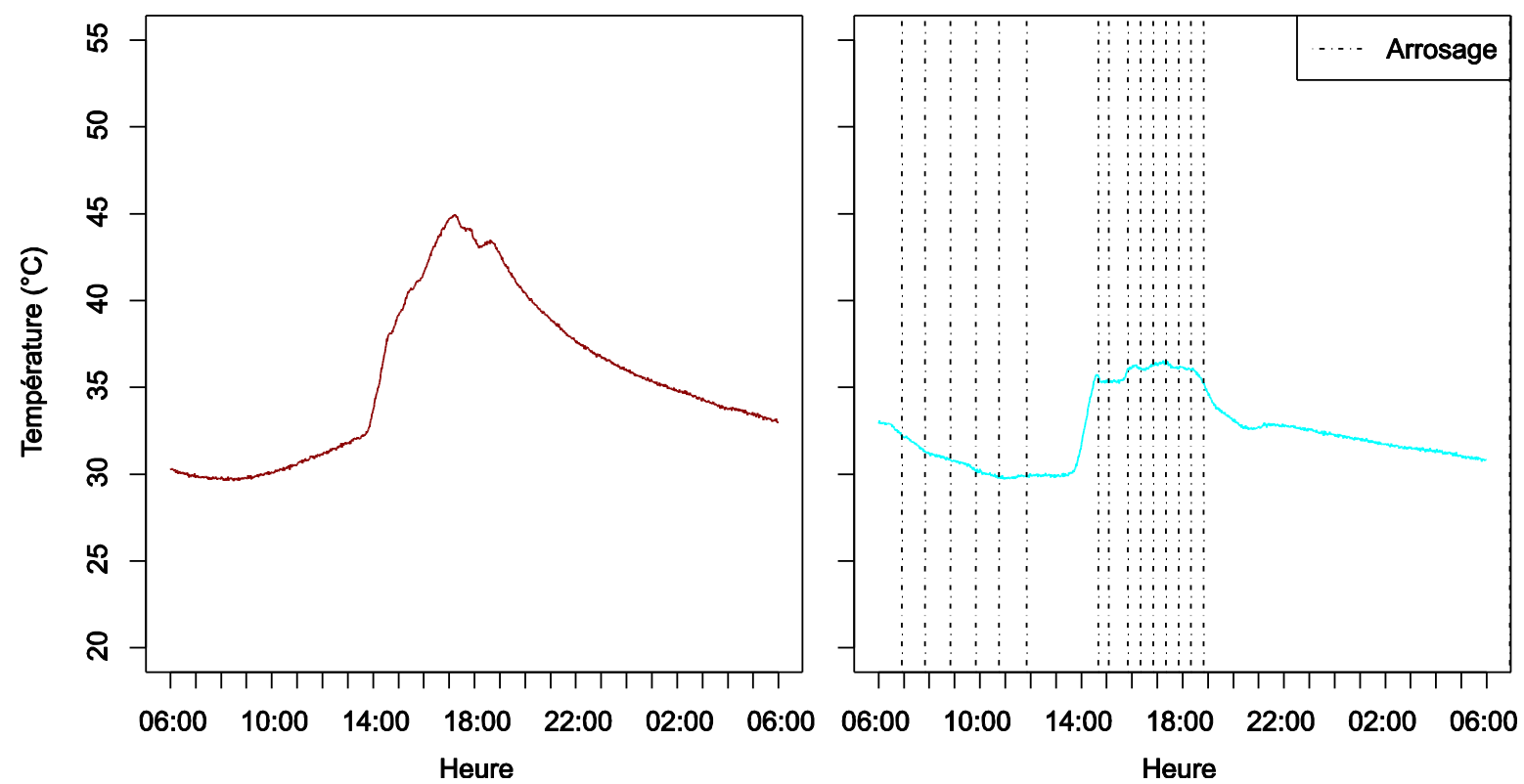

Figure 7 : Température de la chaussée mesurée à $5 \mathrm{~cm}$ de profondeur

(à gauche : 21 juillet 2013 ; à droite : 22 juillet 2013)

Alors que la température oscille entre $30^{\circ}$ et $45^{\circ} \mathrm{C}$ le 21 juillet, elle ne dépasse pas les $37^{\circ} \mathrm{C}$ le 22 juillet. De la même façon que pour les températures de surface, la température minimale est atteinte pendant plusieurs heures lors de l'arrosage. Par ailleurs, le déclenchement de l'arrosage le matin provoque une accélération de la décroissance en température. Compte tenu des températures observées entre minuit et $6 \mathrm{~h} 30$, on estime que la réduction obtenue atteint les $5^{\circ} \mathrm{C}$. Ensuite, alors 
que l'ensoleillement provoque une croissance de température jusque $17 \mathrm{~h} 15$ le 21 juillet, cette phase est interrompue par le premier arrosage vers $14 \mathrm{~h} 30$ le 22 juillet. La température atteint alors un plateau qui dure jusque la fin de l'ensoleillement.

L'arrosage a donc des effets analogues sur la température à $5 \mathrm{~cm}$ de profondeur que pour celle de surface. Les températures maximales et minimales sont réduites de plusieurs degrés et la température minimale est maintenue pendant plusieurs heures. L'arrosage empêche la température d'augmenter entre $15 \mathrm{~h}$ et $18 \mathrm{~h} 30$. Le pic journalier atteint en conditions sèches est ainsi remplacé par un plateau environ $8^{\circ}-9^{\circ} \mathrm{C}$ plus frais.

\subsection{Flux de chaleur à $5 \mathrm{~cm}$ de profondeur}

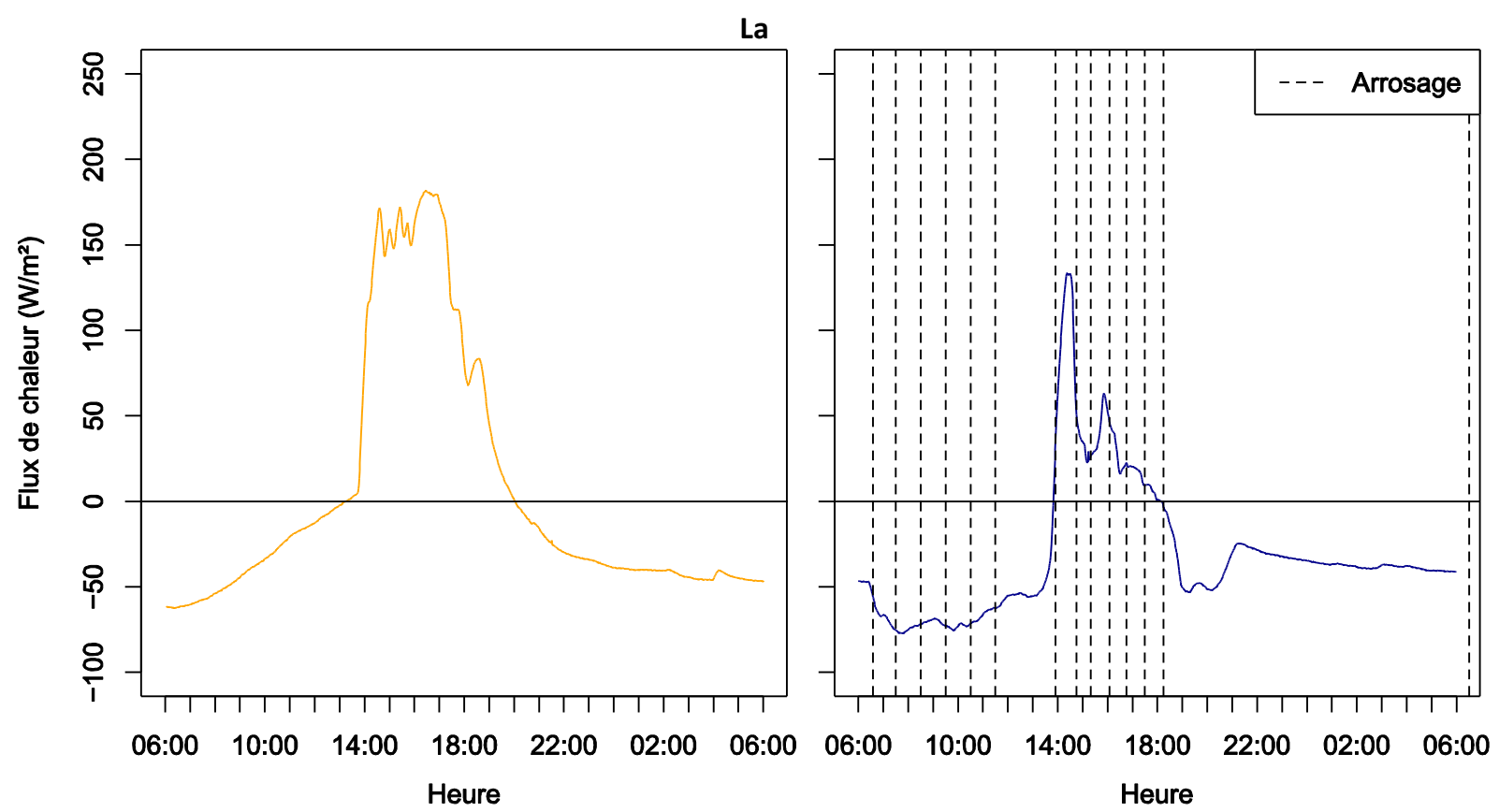

Figure 8 présente les mesures enregistrées aux mêmes dates par le capteur de chaussée installé à 5 $\mathrm{cm}$ de profondeur. Comme pour la Figure 5, les droites verticales en tirets-pointillés le 22 juillet représentent les passages de la laveuse et les droites en pointillés du 21 juillet représentent le début et la fin de l'ensoleillement de la chaussée au-dessus du capteur. Comme précisé précédemment, les valeurs positives correspondent à un stockage de chaleur, alors que les valeurs négatives traduisent une restitution de chaleur vers l'atmosphère. 

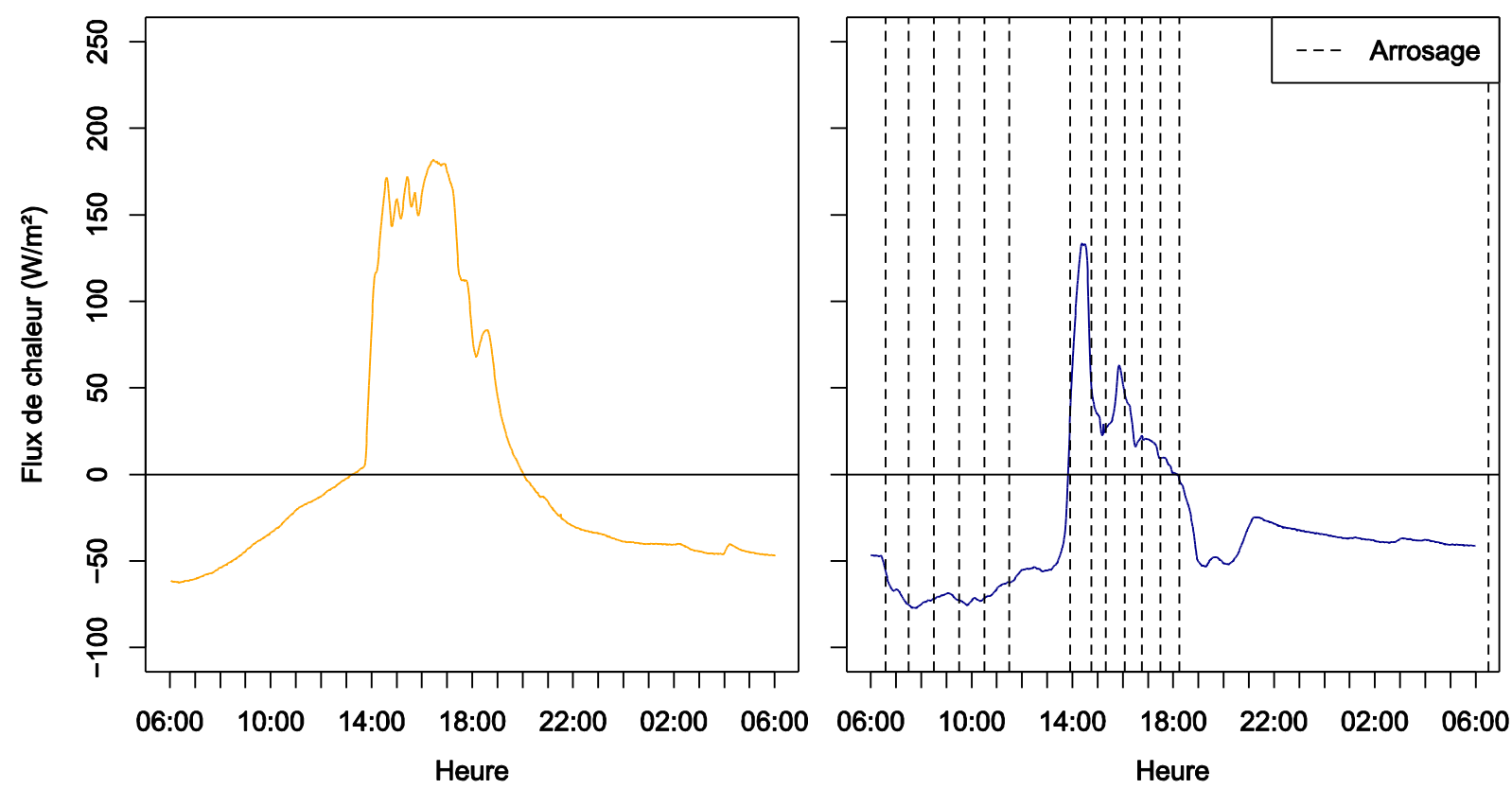

Figure 8 : Flux de chaleur mesuré à $5 \mathrm{~cm}$ de profondeur rue du Louvre

(à gauche : 21 juillet 2013 ; à droite : 22 juillet 2013)

Le comportement du flux de chaleur à $5 \mathrm{~cm}$ de profondeur est très similaire à celui des températures de surface. Le flux de chaleur évolue dans un intervalle similaire en conditions sèches et arrosées, à savoir entre -60 et $190 \mathrm{~W} / \mathrm{m}^{2}$. Par contre, la valeur minimale est plus faible $\left(-15 \mathrm{~W} / \mathrm{m}^{2}\right.$ environ) et maintenue pendant plusieurs heures lors de l'arrosage. L'allure globale, celle d'une courbe en cloche le 21 juillet (dont les perturbations dus aux passages nuageux sont plus visibles), est fortement perturbée par l'arrosage.

Le matin, le premier arrosage entraîne une brusque chute du flux de $-50 \mathrm{~W} / \mathrm{m}^{2}$ à $-76 \mathrm{~W} / \mathrm{m}^{2}$. L'aprèsmidi pendant l'ensoleillement, l'arrosage maintient le flux en deçà de $50 \mathrm{~W} / \mathrm{m}^{2}$, sauf entre $15 \mathrm{~h} 45$ et $16 \mathrm{~h}$ où on observe un pic secondaire qui fait suite à une longue interruption de l'arrosage. Comme pour les températures de surface, le pic principal est atteint quelques minutes avant le premier arrosage de l'après-midi. Enfin, un "puits » de flux est visible entre $18 \mathrm{~h} 30$ et $21 \mathrm{~h}$, lié lui aussi au passage à l'ombre puis au séchage de la chaussée au-dessus du capteur. Enfin, une fois sèche à $21 \mathrm{~h}$, la chaussée libère moins d'énergie qu'en conditions sèches.

On constate immédiatement les effets de l'arrosage sur le flux de chaleur à $5 \mathrm{~cm}$ de profondeur : flux maximal fortement réduit, flux minimal maintenu pendant plusieurs heures malgré des flux avant arrosage plus élevés, division par plus de deux du flux absorbé par la chaussée pendant son ensoleillement.

Les observations de l'effet de l'arrosage sur le flux à $5 \mathrm{~cm}$ de profondeur sont donc analogues à celles faites sur les températures de surface. Compte tenu des mécanismes de diffusion thermique en jeu, cela est attendu et participe à renforcer nos observations et analyses.

\subsection{Conclusions sur les effets thermiques de l'arrosage}

L'analyse de ces paramètres nous permet d'affirmer que l'effet de l'arrosage sur le comportement thermique des surfaces arrosées est clair et très significatif. Les températures de surface et en profondeur ainsi que le flux de chaleur sont très fortement réduits. L'arrosage est particulièrement efficace pendant l'ensoleillement, lorsque la chaussée emmagasine de l'énergie. C'est également la période pendant laquelle l'évaporation devrait être la plus forte. Cependant, l'effet pendant les périodes d'ombre reste significatif.

On en conclut que l'arrosage pendant l'ensoleillement permet d'éviter aux matériaux de stocker de l'énergie solaire, tandis que l'arrosage nocturne peut permettre de déstocker de l'énergie déjà 
accumulée pendant les jours précédents. Ces effets devraient se répercuter sur les échanges entre les matériaux et l'atmosphère et devraient donc réduire le réchauffement qu'ils provoquent.

\section{EFFETS MICROCLIMATIQUES}

\subsection{Méthode d'analyse}

Pour déterminer les effets microclimatiques de l'arrosage, les travaux précédents menés au Japon ou en France s'appuient sur des comparaisons directes entre les mesures d'un site arrosé et celles d'un site témoin. Ceci est notamment le cas de Bouvier et al. [5], Takahashi et al. [7], Yamagata et al. [8] ainsi que Kinouchi et Kanda [9]. Cette approche suppose implicitement que la différence de mesure pour un paramètre donnée entre le site expérimental et le site témoin est constante au cours de la journée et égale à zéro. Tout écart par rapport à cette valeur est ainsi identifié comme effet de l'arrosage. On considère donc par la suite la différence observée entre la station expérimentale et la station témoin $\left(S=S_{\text {exp }}-S_{\text {tém }}\right)$.

Afin de tenir compte des variations naturelles d'un jour témoin à un autre, on s'intéresse non pas aux mesures brutes, mais à une représentation statistique de celles-ci. Dans les graphiques à suivre, les courbes continues représentent la moyenne observée et les courbes en pointillées représentent l'intervalle de confiance à $95 \%$ de la différence des moyennes des jours arrosés et des jours témoins.

Chaque journée est découpée de $6 \mathrm{~h}$ du matin à 5 h59 le lendemain matin afin d'avoir à disposition une période de $24 \mathrm{~h}$ qui suit le début de l'arrosage.

\subsection{Résultats}

La Figure 9 présente les profils statistiques moyens d'une journée témoin et d'une journée arrosée rue du Louvre pour la température de l'air, l'humidité relative et la température moyenne de rayonnement.

Une lecture rapide révèle que l'arrosage entraîne :

- un rafraîchissement pour la température de l'air et la température moyenne de rayonnement (la courbe bleue est toujours en dessous de la courbe rouge). Son amplitude est variable selon l'heure de la journée ;

- une augmentation de l'humidité de l'air, également d'amplitude variable selon l'heure de la journée.

Moyenné sur 24 heures, l'écart pour la température de l'air est de $-0,20^{\circ} \mathrm{C}$, de $+1,3 \% \mathrm{HR}$ pour l'humidité relative et de $-0,36^{\circ} \mathrm{C}$ pour la température moyenne de rayonnement. Ces écarts quotidiens sont significativement statistiques par rapport à l'incertitude de la différence entre la moyenne quotidienne des jours témoins et celle des jours arrosés.

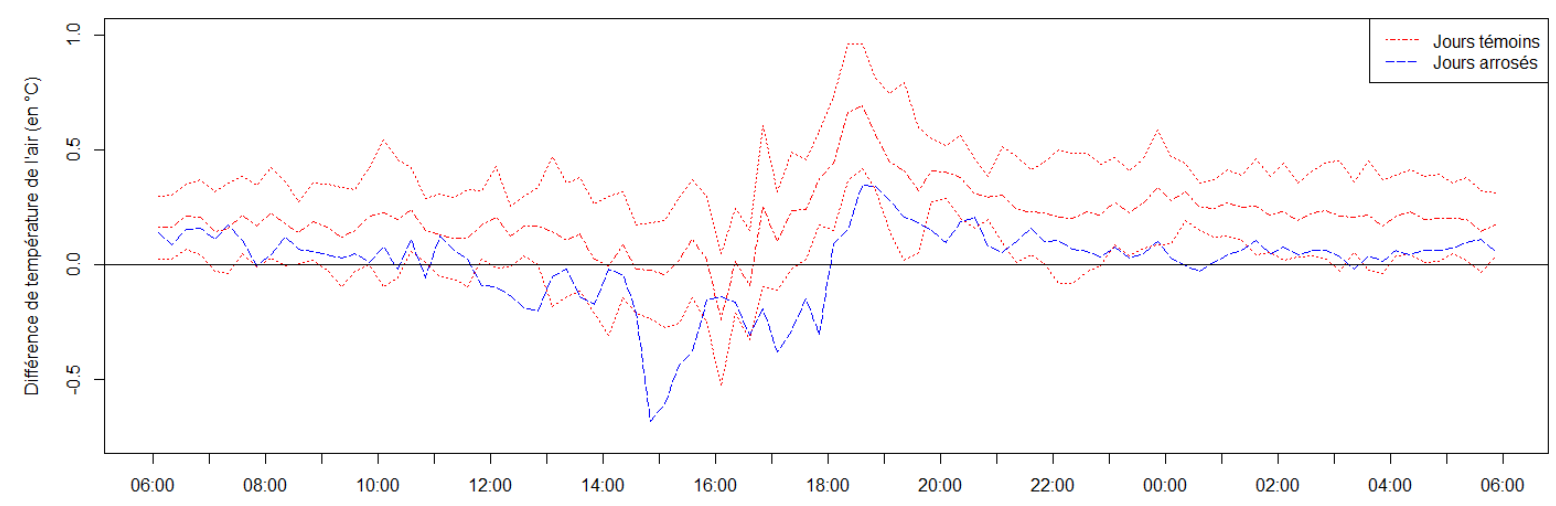



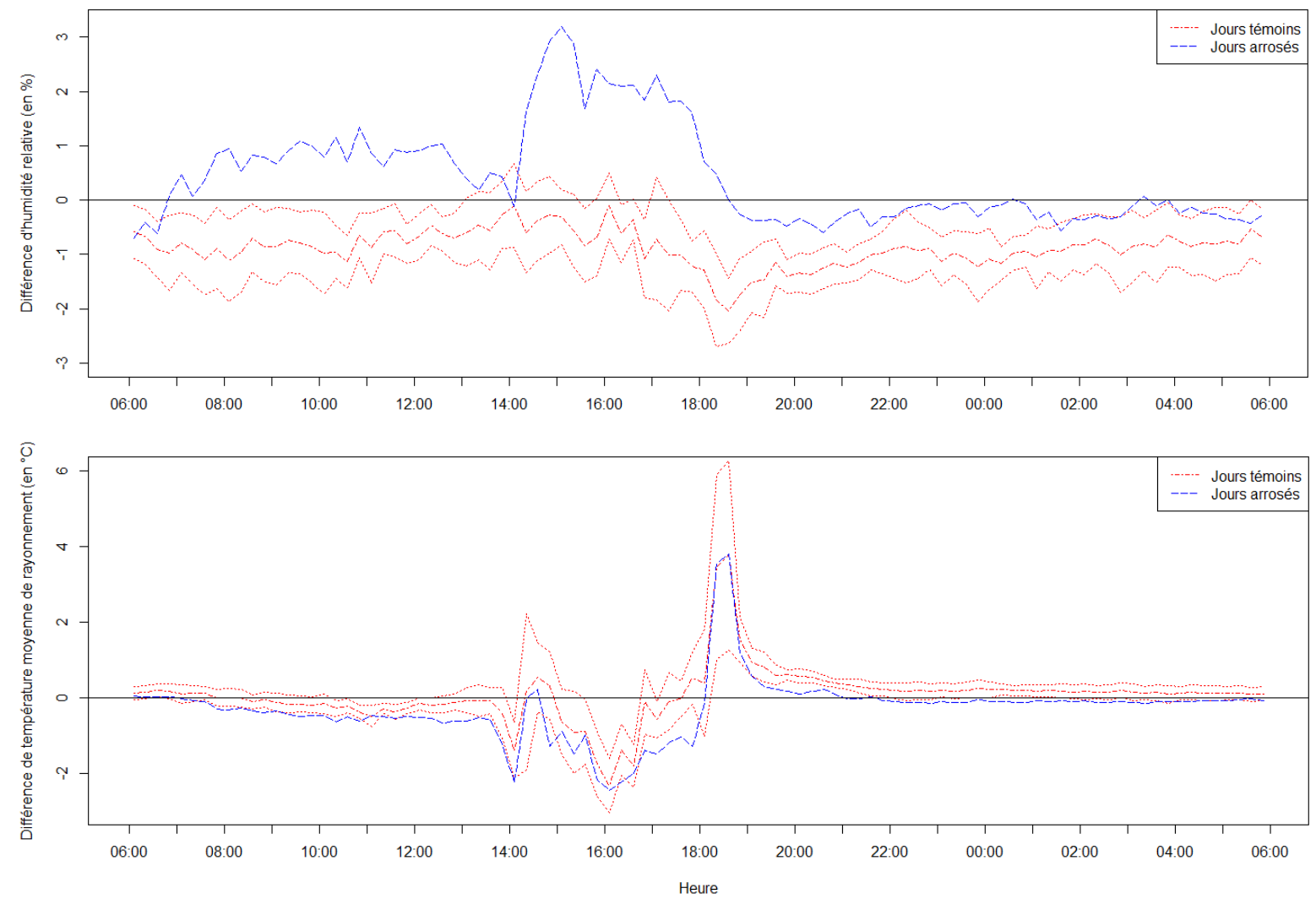

Figure 9 : Différence de la température de l'air (haut), de l'humidité relative (milieu) et de la température moyenne de rayonnement (bas) entre les stations rue du Louvre les jours témoins (rouge) et arrosés (bleu). L'intervalle de confiance à $95 \%$ de la différence des deux courbes continues est également tracé en pointillés.

Les écarts maximaux entre les profils témoins et arrosés atteints au cours de la journée sont de :

- $\quad-0,68^{\circ} \mathrm{C}$ pour la température de l'air ;

- $\quad+3,5 \% H R$ pour l'humidité relative ;

- $\quad-1,79^{\circ} \mathrm{C}$ pour la température moyenne de rayonnement.

Une lecture plus détaillée, tenant compte des intervalles de confiance à $95 \%$ de la différence des moyennes des journées témoins et arrosées, rend compte que les effets moyens observés ne sont pas significatifs à tout moment de la journée (la courbe bleue est comprise entre les deux courbes rouges en pointillés). Alors que l'effet sur l'humidité relative est plus souvent significatif en journée, pendant l'arrosage, l'effet sur les températures est plus souvent significatif la nuit, après l'arrosage. 

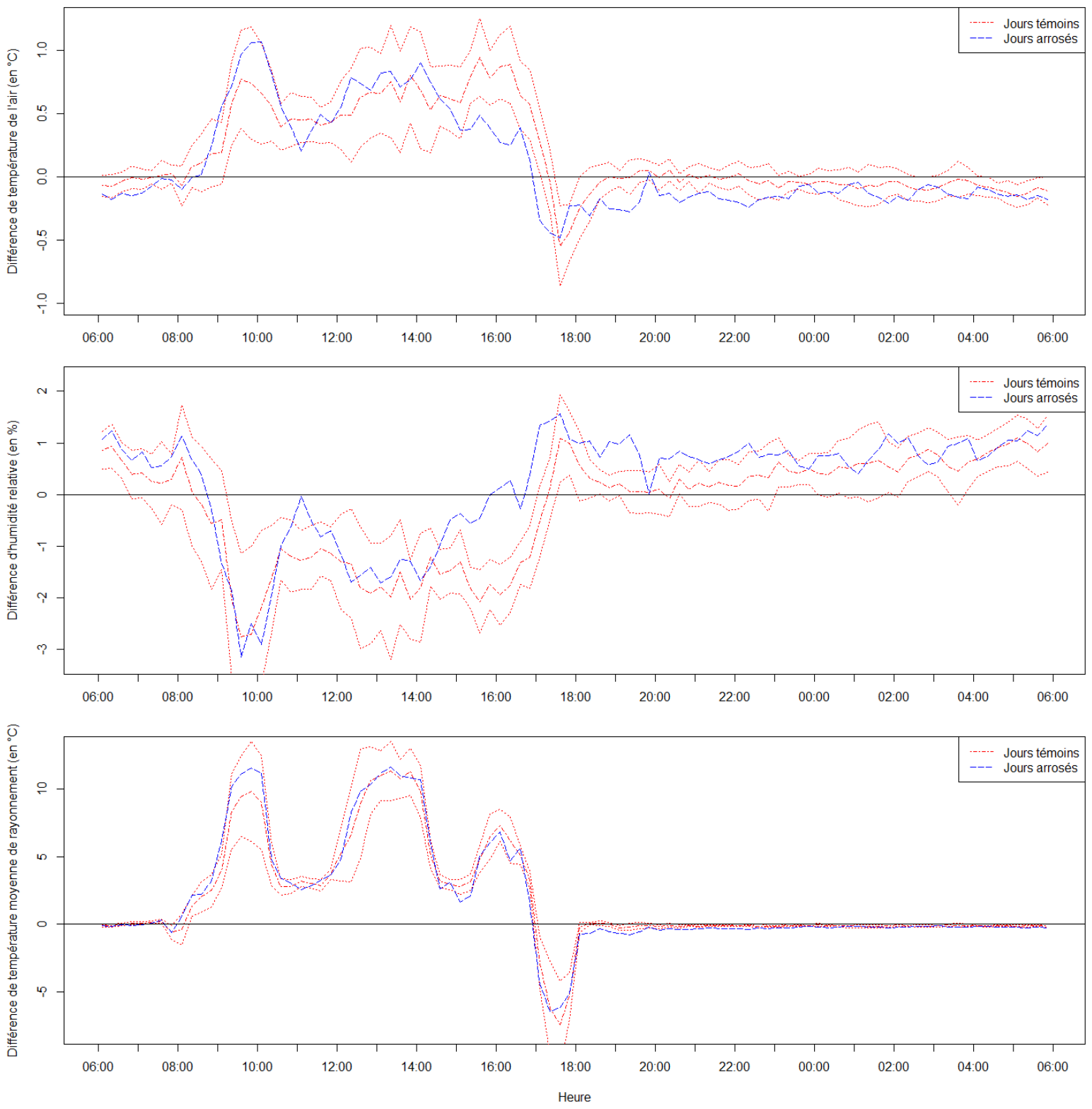

Figure 10 : Différence de la température de l'air (haut), de l'humidité relative (milieu) et de la température moyenne de rayonnement (bas) entre les stations de Belleville les jours témoins (rouge) et arrosés (bleu). L’intervalle de confiance à $95 \%$ de la différence des deux courbes continues est également tracé en pointillés.

En raison du vandalisme de la station témoin de la rue du Louvre, cette analyse tient uniquement compte de la période du 2 au 19 juillet et du 19 août au 4 septembre 2013. II manque ainsi les journées arrosées les plus chaudes que furent les 22 et 23 juillet, $1^{\text {er }}$ et 2 août et le 5 septembre, c'est-à-dire les journées pour lesquels l'effet de l'arrosage devrait être maximal.

Ceci n'est pas le cas des stations situées à Belleville, dans les rues Lesage et Ramponeau, qui ont fonctionné sans interruption sauf entre le 22 et 25 juillet en raison d'un défaut d'alimentation électrique. Les mêmes courbes issues de ces stations sont présentées Figure 10.

En procédant de façon analogue à la rue du Louvre, on remarque :

- un rafraîchissement des températures de l'air et moyenne de rayonnement à partir de 14h30. Son amplitude est également variable au cours de la journée ;

- une augmentation de l'humidité relative de l'air.

Les écarts maximaux entre les profils sont de : 
- $-0,58^{\circ} \mathrm{C}$ pour la température de l'air ;

- $\quad+1,5 \% \mathrm{HR}$ pour l'humidité relative ;

- $-2,94^{\circ} \mathrm{C}$ pour la température moyenne de rayonnement.

De la même façon, les écarts moyennés sur 24 heures sont de $-0,10^{\circ} \mathrm{C}$ pour la température de l'air, de $+0,45 \% \mathrm{HR}$ pour l'humidité de l'air et de $+0,09^{\circ} \mathrm{C}$ pour la température moyenne de rayonnement. En revanche, l'écart quotidien en température moyenne de rayonnement $n$ 'est pas statistiquement significatif.

Une lecture plus détaillée, tenant compte des intervalles de confiance à $95 \%$ de la différence des moyennes des journées témoins et arrosées, rend compte que les effets moyens observés sont moins souvent significatifs que rue du Louvre. Par ailleurs, l'effet pour les trois paramètres est plus souvent significatif la nuit.

L'effet maximal sur la température ou l'humidité relative est plus faible pour Belleville, mais celui sur la température moyenne de rayonnement est sensiblement plus fort. Toutefois, on constate en général des effets moins importants et moins souvent significatifs pour le site de Belleville que pour la rue du Louvre. Plutôt que la différence d'orientation des deux rues, c'est le plus faible arrosage de la rue Lesage en proportion de sa largeur totale qui est retenu comme facteur explicatif de ce constat. En effet, rue du Louvre, $100 \%$ de la largeur de la rue est arrosée (chaussée + trottoirs), alors que ce n'est que $30 \%$ de la rue Lesage (chaussée uniquement). Qui plus est, le positionnement sur les trottoirs des stations (et des piétons) les rend plus sensible à l'arrosage de ces surfaces.

Le rôle de l'orientation joue surtout sur la répartition de l'énergie solaire reçue de part et d'autre de la chaussée. Alors que rue du Louvre, chaque trottoir reçoit à peu près la même quantité d'énergie au cours de la journée, le trottoir Nord de la rue Lesage en reçoit sensiblement plus que le trottoir Sud. II pourrait donc être intéressant d'arroser plus la moitié Nord de la rue que la moitié Sud pour maintenir le sol humide et garantir un rafraîchissement évaporatif tout au long de la journée.

\section{CONCLUSION}

Cet article s'est intéressé à l'analyse des effets thermiques et microclimatiques de l'arrosage de deux sites parisiens : I'un situé rue du Louvre, orientée Nord-Sud; l'autre situé rue Lesage, orientée EstOuest. La totalité de la rue du Louvre (chaussée et trottoir) et la chaussée de la rue Lesage étaient arrosées.

L'analyse des effets thermiques de l'arrosage de chaussée en période caniculaire a révélé des effets rafraîchissants significatifs : jusqu'à $15^{\circ} \mathrm{C}$ de réduction de la température de surface et jusqu'à $9^{\circ} \mathrm{C}$ de réduction de la température à $5 \mathrm{~cm}$ de profondeur de la chaussée ainsi qu'un flux de chaleur dans la chaussée réduit de plus de moitié.

L'analyse statistique des effets microclimatiques nous permet d'affirmer que l'arrosage est responsable d'un rafraîchissement sensible dont l'effet sur le confort piéton n'est pas compensé par l'augmentation en humidité relative. L'effet important sur la température moyenne de rayonnement de l'arrosage traduit la forte influence de la température du sol sur le confort thermique du piéton.

En revanche, même si ce n'est pas le cas de l'effet maximal sur la température moyenne de rayonnement, les effets sur le site de Belleville sont moins importants et moins souvent significatifs que rue du Louvre. Ceci est interprété comme étant dû à la proportion d'arrosage nettement plus faible rue Lesage que rue du Louvre. Arroser uniquement la chaussée est donc moins efficace pour obtenir un rafraîchissement que l'arrosage de toute la largeur de la rue. Qui plus est, compte tenu de la répartition de l'énergie solaire, l'arrosage de la moitié Sud d'une rue Est-Ouest devrait être moins intéressant que celui de sa moitié Nord. II serait intéressant d'explorer la validité de cette hypothèse en arrosant uniquement le trottoir Nord d'une rue Est-Ouest.

L'absence de journées caniculaires en 2013, en plus des indisponibilités de matériel subies lors des jours les plus chauds, n'a pas permis de faire de mesures dans les conditions d'utilisation envisagées 
pour le rafraîchissement urbain par arrosage de l'espace public. Une nouvelle campagne d'essais a été réalisée pendant l'été 2014. Malheureusement seulement deux journées d'arrosage ont eu lieu en raison des conditions météorologiques. Une nouvelle campagne est prévue pour l'été 2015 et devrait permettre de renforcer les observations faites en 2013 et 2014 ainsi que de tirer profit des retours de cette campagne, notamment pour tester un protocole d'arrosage optimisé moins consommateur d'eau.

\section{BIBLIOGRAPHIE}

[1] D. Li and E. Bou-Zeid, "Synergistic Interactions between Urban Heat Islands and Heat Waves: The Impact in Cities Is Larger than the Sum of Its Parts," J. Appl. Meteorol. Climatol., vol. 52, no. 9, pp. 2051-2064, Sep. 2013.

[2] A. Lemonsu, R. Kounkou-Arnaud, J. Desplat, J. L. Salagnac, and V. Masson, "Evolution of the Parisian urban climate under a global changing climate," Clim. Change, vol. 116, no. 3-4, pp. 679-692, 2013.

[3] Météo-France and APC, "Climate change in Paris." Météo-France, APC, Paris, p. 8 (In French), 2012.

[4] Météo-France and CSTB, "EPICEA Project - Final Report," Paris, France (in French), 2012.

[5] M. Bouvier, A. Brunner, and F. Aimé, "Arrosage nocturne des rues et effets induits sur le rafraîchissement ambiant en cas de canicule. Les expérimentations de la Ville de Paris," Tech. Sci. Méthodes, vol. 108, no. 12, pp. 43-52, 2013.

[6] APUR, "Étude sur le devenir du réseau d'eau non potable - Partie 1 : Analyse et diagnostic," 2010.

[7] R. Takahashi, A. Asakura, K. Koike, S. Himeno, and S. Fujita, "Using Snow Melting Pipes to Verify the Water Sprinkling's Effect over a Wide Area," in NOVATECH 2010, 2010, p. 10.

[8] H. Yamagata, M. Nasu, M. Yoshizawa, A. Miyamoto, and M. Minamiyama, "Heat island mitigation using water retentive pavement sprinkled with reclaimed wastewater," Water Sci. Technol. a J. Int. Assoc. Water Pollut. Res., vol. 57, no. 5, pp. 763-771, Jan. 2008.

[9] T. Kinouchi and M. Kanda, "An Observation on the Climatic Effect of Watering on Paved Roads," J. Hydrosci. Hydraul. Eng., vol. 15, no. 1, pp. 55-64, 1997. 